\title{
Atom Lithography: Fabricating Arrays of Silicon Microstructures Using Self-Assembled Monolayer Resist and Metastable Helium Beam
}

\author{
Jianwu Zhang, Zhongping Wang and Zengming Zhang \\ Department of Physics, University of Science and Technology of China, Hefei, \\ The Centre of Physical Experiments, University of Science and Technology of China, Hefei \\ P.R. China
}

\section{Introduction}

Lithography is a top-down technology to produce sub-micrometer feature sizes for the fundamental research and widespread applications. The conventional optical lithography has been developed to not only make the tiny electronic devices to form the basic circuits in today's computer chips, but also fabricate micro/nano electromechanical systems (MEMS/NEMS). However, the conventional photolithographic techniques have now reached their ultimate spatial resolution because of both the light diffraction and the photoresist restrictions [1]. Alternative techniques for traditional photolithography have been proposed and developed to meet the requirements of a high spatial resolution of sub$100 \mathrm{~nm}$ as well as the capability to fabricate an arbitrary structure and achieve mass production [1-10]. Research teams around the word have been investigating alternative techniques including extreme ultraviolet lithography, electron and ion beam lithography, Xray lithography and atom lithography. Each technique has its own advantages and disadvantages, and no one yet knows which one will be the method of choice for the next generation lithography (NGL).

The arrays of micro- and nanostructures fabricated on silicon substrates have now attracted more attention for its interesting characteristics in applications such as photonics, electronics, optoelectronics and sensing [11-15]. The array properties can be tuned further by varying the geometry, the doping, the periodicity, and the size of the micro- and nanostructures $[11,12]$. These controllable and elaborate arrays are commonly fabricated by conventional optical lithography, X-ray lithography, electron-beam or ion-beam lithography [1,16-18]. Unfortunately, there are inherent limitations to pattern over large areas at nanoscale using these lithographical techniques due to the light diffraction, the long-range inter-particle interactions and the proximity effects. To overcome these limitations, it's necessary to develop new alternative techniques with a shorter wavelength and a thinner resist for traditional optical lithography.

Among the alternative techniques for traditional photolithography, atom lithography based on metastable atoms beam (MAB) and self-assembled monolayers (SAMs) has shown significant potential in fabricating arrays of micro- and nanostructures, which is a major goal in nanoscience and nanotechnology [10,19-29]. Metastable atom is one of the atom's electron is 
excited from ground state to metastable state. In metastable state, instead of immediately decay to ground state, the electron will stay for some time (long compare to usuall short lifetime-excited state) before it decays to its ground state. For example, the metastable helium atoms have natural lifetimes $\geq 20 \mathrm{~ms}$, which is much longer than its typical flight times. The energy stored in metastable noble gas atom can be used to create pattern in a SAM that act as resit. When a metastable noble gas atom strikes the SAM resist, the atom will release energy and it will go to ground state. The energy released will change the characteristic of the SAM resist in radius few angstroms from place where the atom strikes. The atom in ground state will not have any effect toward SAM resist because noble gas atoms are not chemically reactive. The locally change of characteristic of the SAM resist will enable us to do wet chemical etching. This new fabrication technique, which bridges the gap between the bottomup chemical self-assembly techniques and the modern top-down lithography, can overcome the intrinsic resolution limitations of traditional photolithographic techniques $[10,19,20]$. In principle, the atom lithography based on neutral MAB and SAM ideally meets the required conditions for sub-100nm fabrication [10]. MAB with a de Broglie wavelength of less than $0.1 \mathrm{~nm}$ eliminates diffractive resolution limitations, and an ultrathin organic SAM resist with a thickness of 1 3nm ensures the sharpness of the edge profile within the resist. Moreover, the neutral metastable atoms are insensitive to the electric and magnetic fields, and the long-range inter-particle interactions are weak. Therefore, this novel method permits the direct and large area manufacturing of micro- and nanostructures on a silicon wafer, avoiding some inherent complications of electron-beam, ion-beam or photolithography. With these unique advantages, the atom lithography with neutral MAB and high-resolution SAM resist makes it possible to achieve nanolithography and provides a potential way in manufacturing structures at a largescale based on micro- and nanoscale features.

During the past fifteen years, considerable attention has also been given to the investigation of the mechanism of metastable atom lithography and microfabrication with different resolutions on various substrates, such as gold, silicon, silicon oxide, and mica [10,19-29]. Direct evidence of the emission of $\mathrm{H}^{+}$and $\mathrm{CH}_{\mathrm{x}}{ }^{+}$from the SAM on the $\mathrm{Au}(111)$ surface under the irradiation of a metastable helium atom beam $\left(\mathrm{He}^{*}-\mathrm{MAB}\right)$ at thermal energy supports the interpretation that the SAM is damaged through the interaction between the outermost surface of the SAM and the metastable atoms. This damage can cause a characteristic surface change from hydrophobic to hydrophilic or a molecular structural change from condensed to cross-linked, which consequentially affect the etching process in the fabrication of the micro- or nanostructure, resulting in the positive and negative pattern transfers, respectively. The typical micropatterning with sub-100 $\mathrm{nm}$ resolution on a silicon substrate covered with a $\mathrm{SiO}_{2}$ or $\mathrm{Au}$ layer has been achieved successfully $[10,21,22,28,29]$. Most of these studies are conducted through multistep etching processes using the cover layers as intermediate masks. For example, in the $\mathrm{He}^{*}-\mathrm{MAB}$ lithography with dodecanethiol-SAM $[10,21,22]$, a Au/Ti coating layer is used to create an intermediate mask on silicon substrate by the first etching process, and then, the intermediate mask pattern is transferred to silicon substrate by the second etching process. It is very interesting to directly transfer negative and positive patterns of SAM induced by $\mathrm{He}^{*}-\mathrm{MAB}$ into a silicon substrate without metal/oxide coating layer to fabricate arrays of micro- and nanostructures.

Recently, the He*-MAB lithography of Si with SAM, which formed directly on silicon substrate by direct covalent linkage instead of metal/oxide coating layers, has been accomplished by our research team $[19,20]$. The latent image formed in SAM by He*-MAB 
passing through a stencil is directly transferred into the underlaying Si substrate by $\mathrm{KOH}$ etching, which is a single step etching process. Undoubtedly, to realize and understand this direct transfer process has opened a novel way in the practical application of the atom lithography in micro- and nanofabrication of silicon, especially in arrays of micro- and nanostructures of silicon.

In this context, we summarized our experimental results obtained during the near past years in detail in the field of atom lithography, where organosilane SAMs and $\mathrm{He}^{*}-\mathrm{MAB}$ were used to pattern the surface of $\mathrm{Si}(111) / \mathrm{Si}(110) / \mathrm{Si}(100)$ wafer substrates without coating intermediate layer. The principle and procedure of atom lithography used in our experiments were introduced firstly. Then the experimental process and achievements were given. Finally, the problems and perspectives about this new technique were also discussed in brief.

\section{Principle and procedure}

\subsection{The general principle and procedure}

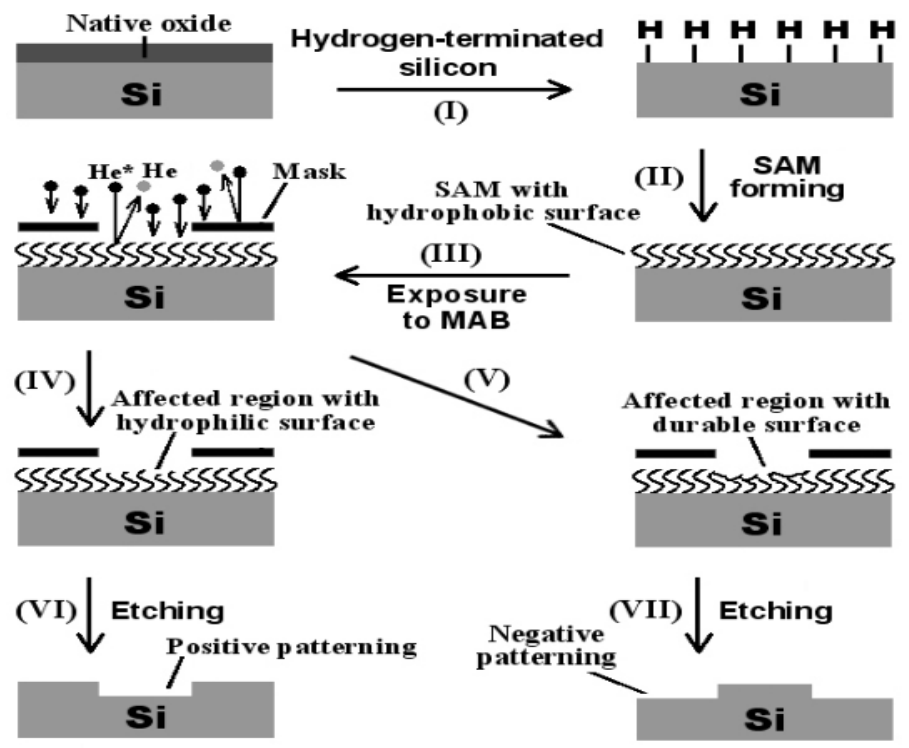

Sheme 1. Shematic illustration of the principle and procedure for the frabrication of periodic arrays of $\mathrm{Si}(111) / \mathrm{Si}(100)$ by $\mathrm{He}^{*}-\mathrm{MAB}$ lithography(excited helium atom:He*; ground-state helium atom: $\mathrm{He})$.

The general principle and process of atom lithography using $\mathrm{He}^{*}-\mathrm{MAB}$ and organosilane SAMs to create the arrays of silicon micro- and nanostructures on a Si substrate were illustrated in Scheme 1. By introducing a hydrogenation process into the pretreatment of silicon substrates (as described in (I) and (II) in Scheme 1), fine organosilanes SAMs was successfully formed as a resist on silicon surface under a controlled argon gas atmosphere at elevated temperature. The chemical modification of non-oxidized silicon surfaces utilizing monolayers could be achieved by neutralizing the silicon surfaces with alkyl chains through 
direct covalent linkage, i.e., silicon-carbon [30-32]. This provided an opportunity to directly use an ultrathin hydrophobic SAM with a thickness of $1 \sim 3 \mathrm{~nm}$ as a super resist on silicon surfaces. The samples with the physical stencil masks were exposed to the irradiation of $\mathrm{He}^{*}-\mathrm{MAB}$ at thermal energy, and the SAM was damaged through the interaction between the outermost surface of the SAM and the metastable atoms (as described in (III) in Scheme 1). When the metastable atoms hit the outermost surface of the SAM on Si substrate, they transferred their internal energy $(\sim 20 \mathrm{eV})$ to the chemisorbed hydrocarbons of SAM molecules and caused chemical changes that might result in either the formation of a durable crossing-linking polymerization material or the loss of hydrophobicity of the SAM (as indicated in (IV) and (V) in Scheme 1). After exposure, the pattern was transferred into a latent pattern in SAM. The SAM exhibited the positive-tone and negative-tone sensitivity due to largely different dosage of MAB and the length of the alkyl chains of SAM molecule. The negative pattern implied the polymerization of SAM molecules induced by more $\mathrm{He}^{*}$ MAB irradiation dose. Meanwhile, the positive pattern indicated the hydrophilic region of SAM induced by less He*-MAB irradiation dose. Furthermore, the longer alkyl chains were easier to undergo cross-linking polymerization than the shorter alkyl chains under the $\mathrm{He}^{*}$ MAB irradiation. Consequently, these could affect the etching process in the fabrication of the micro- or nanostructure (i.e., the hydrophilic exposure region was easier to etch using $\mathrm{KOH}$ solution than the hydrophobic unexposed region, whereas the cross-linking polymerization region was more durable to $\mathrm{KOH}$ etching than the hydrophobic unexposed region), resulting in the positive and negative pattern transfers (as described in (VI) and (VII) in Scheme 1), respectively.

The mechanism for the contrast inversion, i.e., the competition between the loss of hydrophobicity and the cross-linking polymerization of SAM molecules determined the polarity of the pattern transfer process, has been confirmed previously $[19,20]$. In our experiments, the lithographic patterns were obtained in samples with a relatively large area of about $2 \mathrm{~cm}^{2}$. In these areas, the mask pattern was reproduced with high fidelity, and the repetition of lithographic patterns was consistent in different runs. In order to obtain better contrast patterns, we carefully optimized the experimental parameters. After etching, the resulting arrays fabricated on the silicon substrate were clearly observed under the optical microscope.

\subsection{Mechanism of forming SAMs on Si substrates}

The mechanism of forming SAMs on Si substrates was illustrated in details in Scheme 2. The p-type $\operatorname{Si}(111) / \operatorname{Si}(110) / \operatorname{Si}(100)$ wafers with a native oxide layer (p-type $\operatorname{Si}(111): \Phi=100 \mathrm{~mm}$, thickness $=380 \mu \mathrm{m}, \rho=10 \sim 20 \Omega \mathrm{cm}$; n-type $\operatorname{Si}(110)$ : $\Phi=100 \mathrm{~mm}$, thickness $=300 \mu \mathrm{m}, \rho=1 \sim 5 \Omega \mathrm{cm}$; p-type $\operatorname{Si}(100): \Phi=100 \mathrm{~mm}$, thickness $=300 \mu \mathrm{m}, \rho=10 \sim 20 \Omega \mathrm{cm}$ ) were used and cut into pieces of $10 \mathrm{~mm} \times 20 \mathrm{~mm}$ as Si substrates. The Si substrates were first rinsed using toluene, ethanol, and deionized water sequentially, then immersed into a $2 \mathrm{vol} . \%$ aqueous HF solution for $5 \sim 10 \mathrm{~min}$ to remove the native oxide layer and hydrogenate the silicon substrates, and finally dried. Protected from air by a flowing argon gas, the Si substrates were immersed into a 10 40mM organosilanes (octadecyltrichlorosilane, 18C-long-chain hydrocarbon molecules, ODTS; dodecyltrichlorosilane, 12C-long-chain hydrocarbon molecules, DDTS; hexycyltrichlorosilane, 6C-long-chain hydrocarbon molecules, HTS) toluene solution at room temperature for $24 \sim 50$ hours, and then rinsed in toluene and dried at $100^{\circ} \mathrm{C}$ for 30 80 min. Thus, through direct covalent linkage, i.e., silicon-carbon, fine organosilanes 
SAMs were formed as a resist on silicon surface by introducing a hydrogenation process into the pretreatment of silicon wafer and a controlled argon atmosphere at elevated temperature $[19,20,30]$.

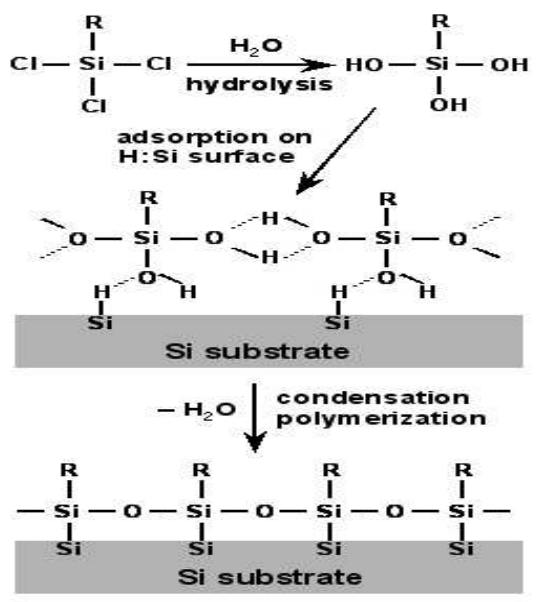

Sheme 2. The illustration of the mechanisam of forming SAMs on Si substrates.

\subsection{Exposure to MAB}

The experimental setup for the sample exposure to $\mathrm{He}^{*}-\mathrm{MAB}$ was depicted in Scheme 3. The Si substrate with organosilane SAMs was covered by a physical mask with TEM Cu grids. As shown in the inset of Scheme 3, six TEM grids were well distributed on a nickel sheet (with a thickness of $0.1 \mathrm{~mm}$ and a square area of $1 \mathrm{~cm} \times 2 \mathrm{~cm}$ ) and fixed over the opening holes with a diameter of $2 \mathrm{~mm}$ using Ag conductive paste. The assembly was immediately inserted into the sample chamber of the MAB source (as shown in Scheme 3) and exposed to $\mathrm{He}^{*}$ MAB for 5 120 min, producing latent patterns in organosilanes SAM on Si substrates. The exposure experiments were performed with the existing apparatus depicted in Scheme 3. The $\mathrm{He}^{*}$ source was operated in a dc discharge mode, and a stable pressure $\left(\sim 5 \times 10^{-7} \mathrm{~Pa}\right.$ for the ultimate pressure and $\sim 3 \times 10^{-2}$ Pa for the working pressure) of chambers was retained by turbo-molecule pump (T.M.P) vacuum systems. A transverse deflecting voltage of $600 \sim 800$ $\mathrm{V}$ was applied along the beam line to eliminate ions or electrons in the beam, which otherwise damage the SAM during exposure. Through a skimmer opening of $\sim 0.7 \mathrm{~mm}$ diameter, $\mathrm{He}^{*}-\mathrm{MAB}$ was directed toward a rotatable thin tantalum $(\mathrm{Ta})$ sheet sample holder at a distance of $26.5 \mathrm{~cm}$. Three tantalum (Ta) plates were arranged triangularly around the rotation axis (shown in the inset of Scheme 3). Two of them were used for installing samples, and the remaining one was used for monitoring the intensity of the primary beam. The thickness of the nickel sheet defined the gap between physical masks and sample surfaces ( 100um), avoiding the mechanical contact that may cause possible artifacts of SAMs. Since the mask almost covered the entire sample, the metastable flux was represented by the metastable-atom-induced electron emission from the Ta plate or from the sample and mask, which was the main content of the induced current. The typical values of the measured current on both the Ta plate and sample were 100 and $50 \mathrm{nA}$, which corresponded to an electron emission rate of $\sim 1 \times 10^{12}$ and $\sim 2 \times 10^{12} \mathrm{~s}^{-1} \cdot \mathrm{cm}^{-2}$, respectively. 


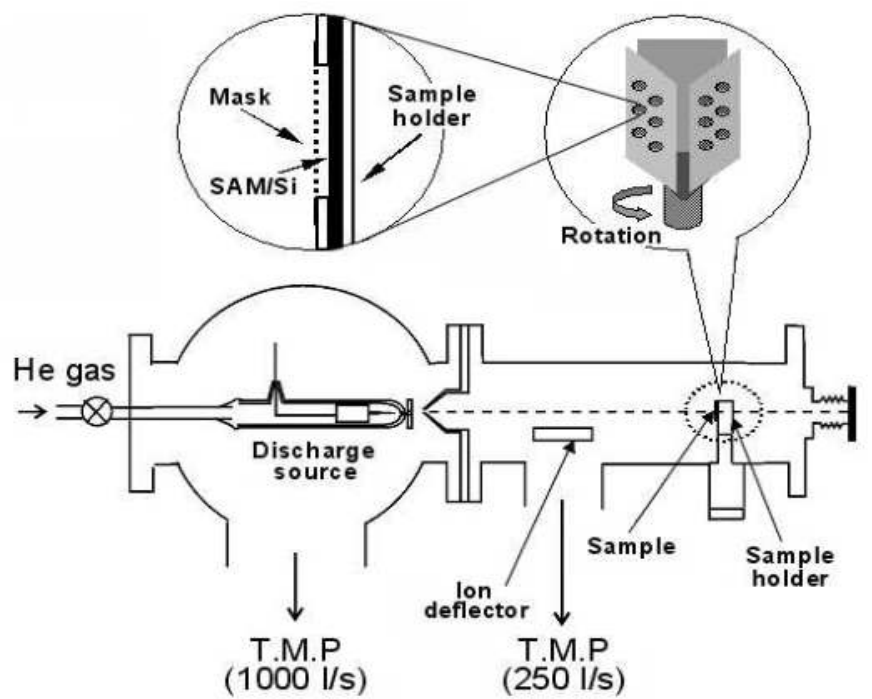

Sheme 3. Shematic setup of the sample exposure to MAB for atom lithography.

During exposure $\mathrm{He}^{*} \mathrm{MAB}$ impacted the surface of SAM formed on silicon substrate after the beam was patterned in transverse direction by the TEM grids. Atoms with kinetic energy smaller than $1 \mathrm{eV}$ do not pass freely through materials. So, the physical mask (i.e., TEM grids) with thickness in the order of sub-micrometers was enough to pattern the $\mathrm{He}^{*}$ MAB. Our experimental results showed good reproducibility with a high fidelity of the patterns of the TEM copper grids and TEM holey carbon grids (shown in figure 1), as well as a good repetition of the lithographic patterns in different runs.

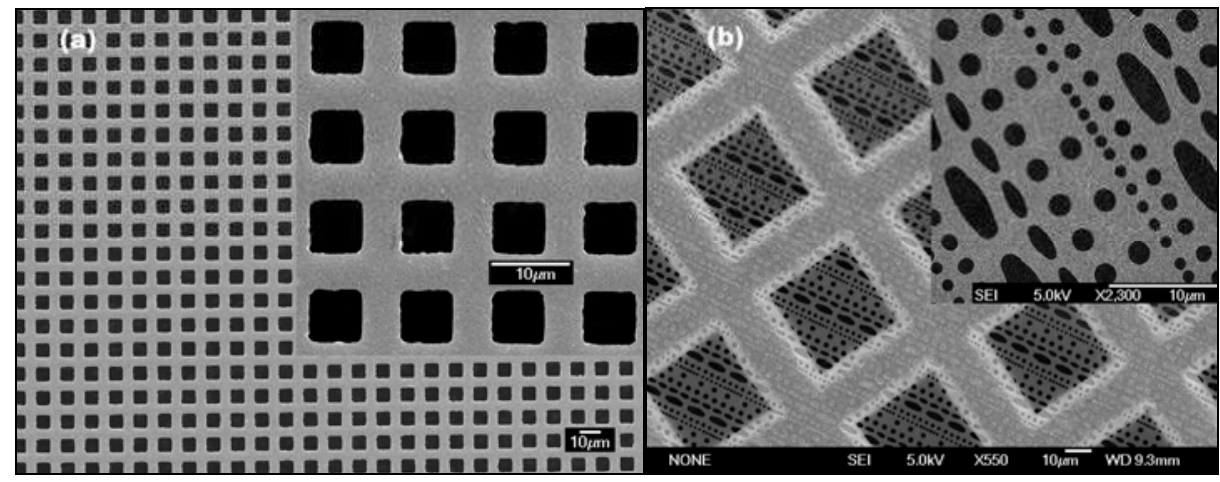

Fig. 1. (a) SEM image of TEM Cu grid used as a mask (Note: the pitch size of patterns is 12.5 um, $5.5 \mathrm{um}$ for wires, and $7.0 \mathrm{um}$ for spaces) and (b) SEM image of TEM holey carbon grid used as a mask (Note: The diameters of the circular holes are about 1, 1.4, and $2 \mu \mathrm{m}$, the bar widths range from 0.5 to $4 \mu \mathrm{m}$, the oval holes with a dimension of $8 \times 2 \mu \mathrm{m}$ and $4 \times 1 \mu \mathrm{m}$ ).

Two kinds of physical masks (i.e., TEM Cu grids and TEM holey carbon grids) were used in our experiments. The SEM images and parameters of the TEM grids were shown in figure 1. 
The bar widths and the hole diameters define the pitch sizes of the patterns. Even though the image of a pattern was in micrometer range, a sharp edge in the tens-of-nanometers range for the resulting microstructures of silicon indicated a potential of nanosized pattern in our experiments. In this sense, actually, the spatial resolution of the patterning in our experiments was not ascribed to TEM grid mask but to the edge resolution.

\subsection{Etching processing and pattern transferring}

After exposure, the samples with latent patterns in SAM were taken out from the vacuum chamber and rapidly dipped into an etching solution in which a magnetic bar was used for stirring, and were etched for 5 60min at room temperature in an aqueous solution of $0.1 \mathrm{M}$ $\mathrm{KOH}$, rinsed by deionized water, and then dried in air. By this single step $\mathrm{KOH}$ etching process, the latent image formed in SAM by $\mathrm{He}^{*}-\mathrm{MAB}$ passing through a stencil was directly transferred into the underlaying Si substrate (as described in (VI) and (VII) in Scheme 1).

For the wet etching of $\mathrm{Si}$ substrate in $\mathrm{KOH}$ solution, the anisotropy of etching rate of silicon have been studied in detail over the passed 20 years. Especially, in the case of the photolithography in MEMS and IC industry, the anisotropy of etching rate of silicon has been investigated completely, and the anisotropy can induce obvious differences of the spatial resolution of patterning of $\mathrm{Si}$ along different crystal orientation. The etching rates for the silicon wafer in the $\mathrm{KOH}$ solution are known to depend strongly on its orientation $\left(\mathrm{Si}+2 \mathrm{OH}^{-}+2 \mathrm{H}_{2} \mathrm{O} \rightarrow \mathrm{SiO}_{2}(\mathrm{OH})_{2}{ }^{2-}+2 \mathrm{H}_{2(\mathrm{~g})}\right.$; etching ratio: $\left.\{110\}>\{100\}>>\{111\}\right)$ [33]. In our experiments, the anisotropic etching process of silicon occurred in the same way, and strongly affected the patterning results including edge step resolution. The sharpest step edge resolution was only $41 \mathrm{~nm}$ with 1 pixel and the average step edge resolution was around $100 \mathrm{~nm}$.

\subsection{Characterization}

AFM images of the samples were taken by a multimode Nanoscope IV atomic force microscope (Veeco Metrology Group, Santa Barbara, CA), operated at ambient conditions. For all images we recorded the retrace direction of the tip using a scan angle of $0^{\circ}$ or $90^{\circ}$. Substrates decorated with organosilanes SAMs were imaged in tapping mode using silicon cantilevers (NanoWorld, Neuchâtel, Switzerland) with a spring constant of $42 \mathrm{~N} \mathrm{~m}^{-1}$. All images were recorded at a rate of $1.0 \mathrm{~Hz}$, and with a pixel resolution of 512. SEM inspection of patterned Si substrates and the masks were carried out with a JSM-6500/SG scanning electron microscope operated at $30 \mathrm{keV}$.

\section{Experimental achievements}

Up to now, we have developed a process successfully, and realized a He*-MAB lithography of $\mathrm{Si}(111) / \mathrm{Si}(110) / \mathrm{Si}(100)$ wafer substrates with SAMs, where the SAMs was formed not on metal/oxide coating layers but directly on silicon substrate. The process of atom lithography using $\mathrm{He}^{*}-\mathrm{MAB}$ and organosilane SAMs to create the arrays of silicon micro- and nanostructures on a Si substrate were illustrated in Scheme 1. The first step was the formation of very fine organosilanes (ODTS, DDTS and HTS) SAMs as a resist on $\mathrm{Si}(111) / \mathrm{Si}(110) / \mathrm{Si}(100)$ surface by introducing a hydrogenation process into the pretreatment of silicon wafer and a controlled argon gas atmosphere at elevated 
temperature. After the development of micro stencil masks for $\mathrm{He}^{*}-\mathrm{MAB}$ exposure to generate latent patterns in SAMs was carried out, the chemical etching process to transfer the latent patterns into the underlying silicon substrate was examined and optimized. By adjusting experimental parameters (i.e., different masks, exposure time, etching time, etc.), various arrays of silicon microstructures were fabricated successfully. The resulting arrays of silicon microstructures on Si substrates were characterized by the AFM/SEM.

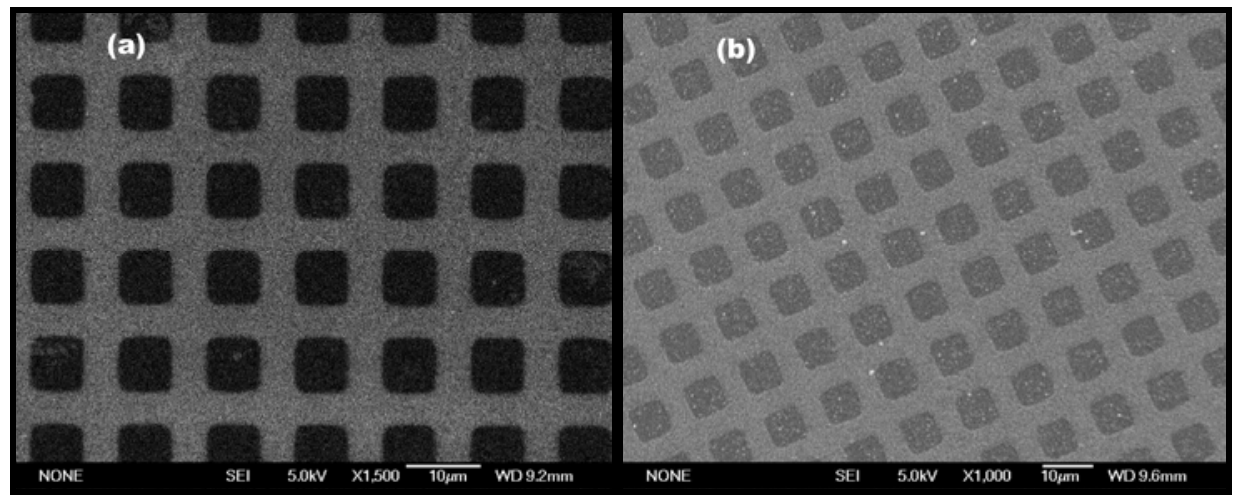

Fig. 2. (a) SEM image of negative patterning (exposure time: $60 \mathrm{~min}$; etching time: $5 \mathrm{~min}$ ) and (b) positive patterning (exposure time: 20min; etching time: $5 \mathrm{~min}$ ) on $\mathrm{Si}(110)$ substrate with the ODTS SAM and DDTS SAM, respectively. Mask: TEM Cu grid.

The SEM images of two samples were displayed in figure 2. The pattern from the stencil mask (as shown in figure 1(a)) was transferred into the $\mathrm{Si}(110)$ substrate, and the resulting micromesa/microwell arrays of silicon fabricated on the $\mathrm{Si}(110)$ substrate were very intact and clear (as shown in figure 2). The preliminary SEM observations revealed that the direct fabrication of the array on the surfaces of $\mathrm{Si}(110)$ substrates by $\mathrm{He}^{*}-\mathrm{MAB}$ and SAMs was viable, and our experimental results indicated that the patterning also appeared on either kind of surface of the $\mathrm{Si}(111)$ or $\mathrm{Si}(100)$ substrate with various organosilane SAM mentioned above. The main experimental results and discussions were given as following.

\subsection{Arrays of $\mathrm{Si}(111)$ microstructures}

The AFM images with the sectional analysis of the square micromesa arrays formed on the Si(111) substrate with ODTS SAM were shown in figure 3, which corresponds to negative patterning with an exposure time of $40 \mathrm{~min}$ and an etching time of $60 \mathrm{~min}$. A square silicon micromesa arrays with a size of $\sim 7 \times 7 \mu \mathrm{m}^{2}$ and a nanoscale edge resolution of $\sim 100 \mathrm{~nm}$ was successfully fabricated. The height of the step was $\sim 30 \mathrm{~nm}$, indicating an etching rate of 0.5 $\mathrm{nm} / \mathrm{min}$. The width of the step edge was 1-3 pixels (41 123 nm). The sharpest step edge had a width of 1 pixel $(41 \mathrm{~nm})$ and a height of $22 \mathrm{~nm}$, indicating a steep sidewall with an aspect ratio of 2:1, which was considered to be the achievable limit in the present case. In fact, after subtracting the effects of the tip shape on the lateral resolution induced by the AFM tip with a $\sim 10 \mathrm{~nm}$ radius of curvature at its end, the real step width should be smaller than the value mentioned above, and the aspect ratio should be larger. The cross sections were not flat, and the rms roughness of the top area of the micromesa and the pedestal base area were $6.2 \mathrm{~nm}$ and $5.8 \mathrm{~nm}$, respectively. This relatively rough characteristic could be ascribed to the 
imperfect SAM formation (i.e., the density of SAM molecules anchored on the Si(111) surface through chemical bonds is not perfectly uniform side by side) on the silicon substrate, which induced a local fluctuation in the etching rate of transferring the latent image of the SAM resist onto the underlying silicon substrate and, consequently, produced a rough surface. Flatter surfaces was obtained using a perfect SAM and under optimal etch conditions. Another characteristic was shown in figure 3, i.e., an upward fringe around the edge of the top of the square micromesas. The etching rates for the silicon wafer in the $\mathrm{KOH}$ solution depended strongly on its orientation [33]. Thus, the etching parallel to the $\operatorname{Si}(111)$ surface proceeded much faster than that perpendicular to the Si(111) surface and undercuts the sidewalls of a micromesa capped with a durable SAM resist that withstands the etching by $\mathrm{KOH}$ solution, leaving a fringe around the micromesa. After the sample was dried, the fringe rolls up or down around the edge of the silicon micromesa. The upward fringe was clearly observed in the 3D image as shown in figure 3(b), suggesting the strong durability of the exposed SAM resist against chemical etching.

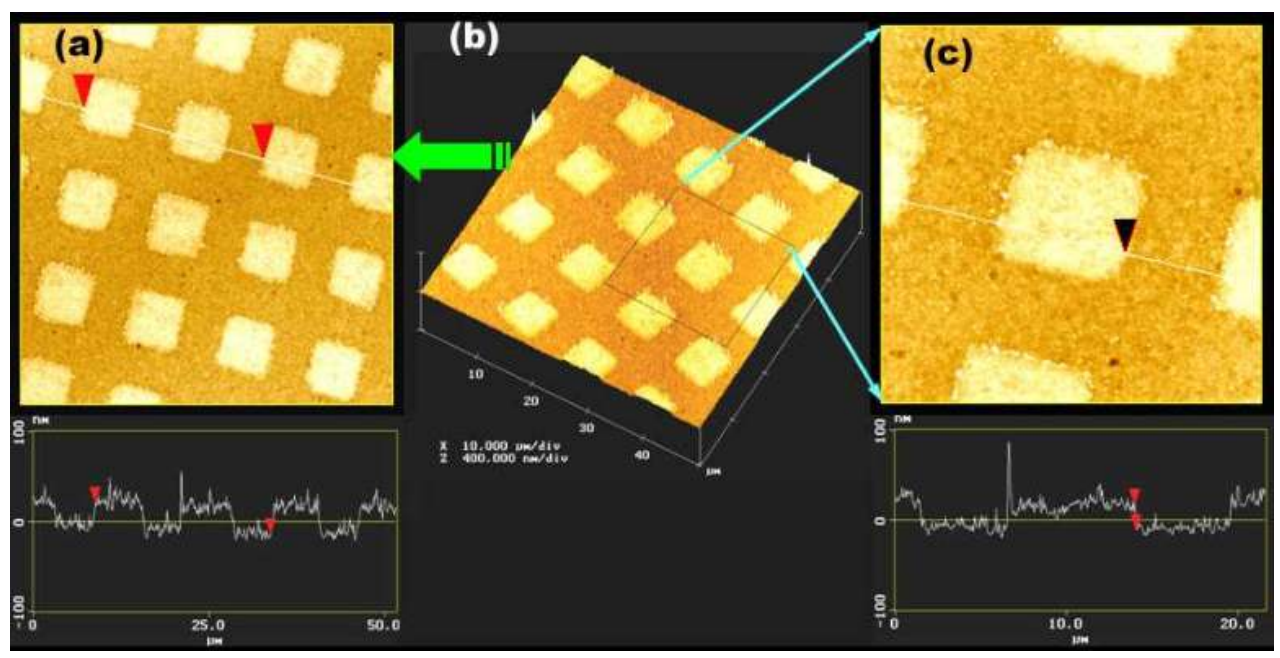

Fig. 3. Tapping-mode AFM images with sectional analysis of square silicon micromesa array created on $\mathrm{Si}(111)$ substrate (Negative patterning, exposure time: 40min; etching time: 60min, SAM: ODTS) (a): 50×50 $\mu \mathrm{m}^{2}$; (b) 3D image; (c): $\left.21.7 \times 21.7 \mu \mathrm{m}^{2}\right)$.

We also obtained a positive pattern within the exposed region with another TEM grid window near the border of the irradiation area. The border region accepted considerably sparser irradiation of He-MAB than the center of He-MAB. Consequently, this exposed border region only changed from hydrophobic to hydrophilic without cross-linking between molecules, which leaded to a weak resistance to chemical etching. Figure 4 showed AFM images and the sectional analysis of the microwell array formed on the $\mathrm{Si}(111)$ substrate, corresponding to the positive patterning. A square silicon microwell array with a size of $\sim 7 \times 7 \mu \mathrm{m}^{2}$ and a depth of $\sim 10 \mathrm{~nm}$ was formed. The topography and line profile of the microwell array indicated a much rougher surface with an rms roughness of $\sim 7 \mathrm{~nm}$. In comparison with the depth of $\sim 10 \mathrm{~nm}$, this large rms value suggested that it was difficult to achieve a perfect positive pattern transfer using the ODTS SAM resist, possibly because the 
hydrophilic area might not be much weaker in resisting the etchant than the unexposed hydrophobic area.

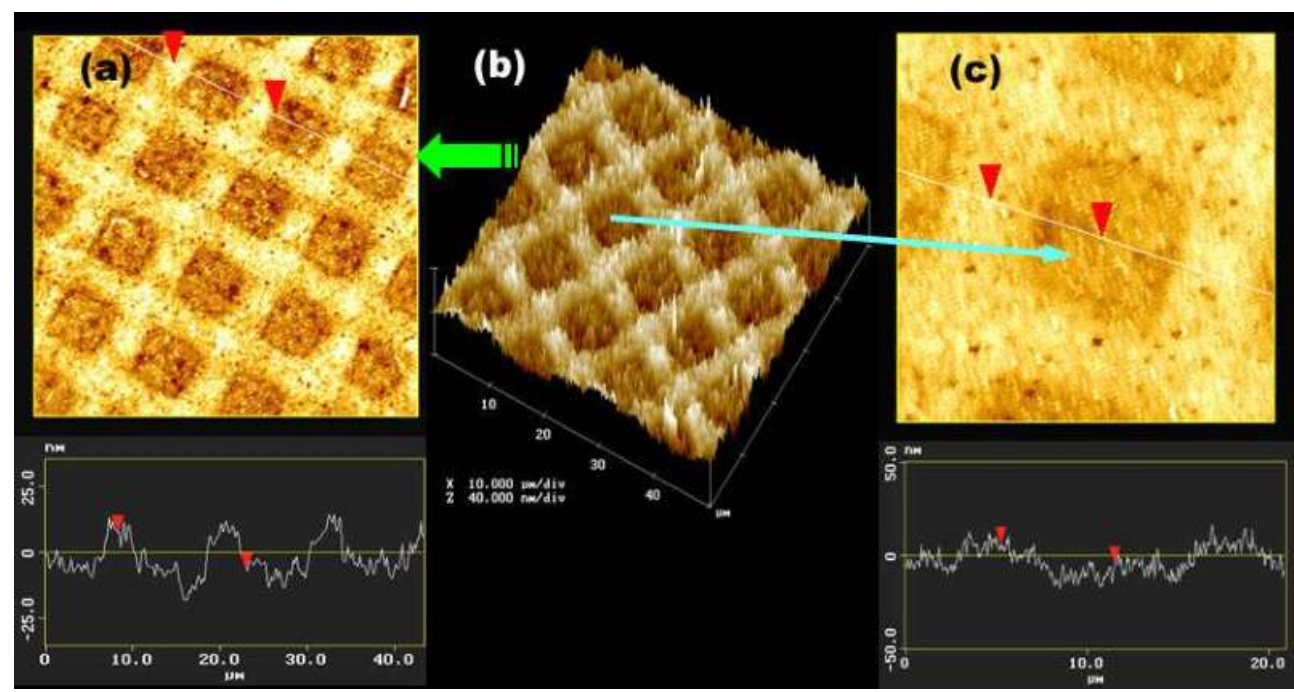

Fig. 4. Tapping-mode AFM images with sectional analysis of square silicon microwell array created on $\mathrm{Si}(111)$ substrate (Negative patterning, exposure time: 40min; etching time: 60min, SAM: ODTS) (a): 50×50 $\mu \mathrm{m}^{2}$; (b) 3D image; (c): $21.7 \times 21.7 \mu \mathrm{m}^{2}$ ).

Since the ODTS SAM resist comprised highly oriented 18C-long-chain hydrocarbon molecules chemically bonded to the silicon substrate, its surface became hydrophobic [30-32]. Previous studies revealed that, under irradiation of He-MAB with a $20 \mathrm{eV}$ internal energy, $\mathrm{C}-\mathrm{H}$ bond scission and $\mathrm{C}-\mathrm{C}$ bond scission occured and a $\mathrm{C}=\mathrm{C}$ double bond could be formed at the outermost surface of the SAM [34-37]. At low doses, breaking of the $\mathrm{C}-\mathrm{H}$ bond caused $\mathrm{H}$ loss, leaving a $\mathrm{C}$-rich film that could be oxidized in air. Consequently, the exposure region was changed from hydrophobic to hydrophilic, leading to a positive pattern [38,39]. At high doses, further degradation of the hydrocarbon molecules caused fragmentation of the carbon chain, and cross-linking polymerization between adjacent fragmented SAM molecules might occur, giving rise to the formation of a highly resistant carbonaceous layer and leading to a negative pattern [36]. In addition, the contamination resist, which was induced by hydrocarbon molecules remaining on the inner wall of the vacuum chambers, remained a concern [40-41]. When the metastable atoms impacted the surface, they transferred their internal energy to these physisorbed hydrocarbon molecules and induced a polymerization change within the exposed region. To clarify whether these resist materials could remain on the surface even after immersion in a $\mathrm{KOH}$ solution, a clean $\mathrm{Si}(111)$ sample without an SAM was used. Only a trace of negative contrast was observed, indicating a negligible contamination effect. In our negative pattern case, the exposure region of the SAM resist became etching-resistant, which might be mainly ascribed to the cross-linking polymerization of the SAM molecules. 


\subsection{Arrays of $\mathrm{Si}(110)$ microstructures}

The AFM images of the square micromesa arrays formed on the Si(110) substrate with ODTS SAM was shown in figure 5 , which corresponded to negative patterning with an exposure time of $20 \mathrm{~min}$ and an etching time of $5 \mathrm{~min}$. A square silicon micromesa array with a micromesa size of $\sim 7 \times 7 \mu \mathrm{m}^{2}$ (e.g., the size of one mesh of TEM stencil mask) was fabricated successfully on the substrate. The average height of the step was $27 \mathrm{~nm}$, indicating an etching rate at $5 \mathrm{~nm} / \mathrm{min}$. The sharpest step edge had a width of 1 pixel $(41 \mathrm{~nm})$ and a height of $26 \mathrm{~nm}$, indicating a steep sidewall with an aspect ratio of 1:1.7. The sectional analysis shown in Figure 5(a) indicated that the rms roughness of the top area of the micromesa and the pedestal base area were $2 \mathrm{~nm}$ and $15 \mathrm{~nm}$, respectively. The flat top area of the micromesas could be observed clearly, suggesting the strong durability of the exposed SAM resist against chemical etching. The flaw pedestal base area of the micromesa was also very conspicuous, and this rough characteristic could be attributed to the imperfect SAM formation on the silicon substrate, which induced a local fluctuation in the etching rate of transferring the latent image in SAM resist onto the underlying silicon substrate and, consequently, produced a rough surface. As described in scheme 1, the negative-tone sensitivity of the SAM might be mainly due to the cross-linking polymerization of ODTS molecules induced by the irradiation of the $\mathrm{He}^{*}-\mathrm{MAB}[19,20]$. In addition, it was noteworthy that the etching time had a notable influence on the production of high-quality patterning. In our practical runs, the following etching times were chosen: 5, 10, and $20 \mathrm{~min}$ with identical exposure times. The sectional analysis of AFM images of these samples indicated that the rms roughness of top area of the micromesa in the case of overetching was bigger than that in the case of underetching.

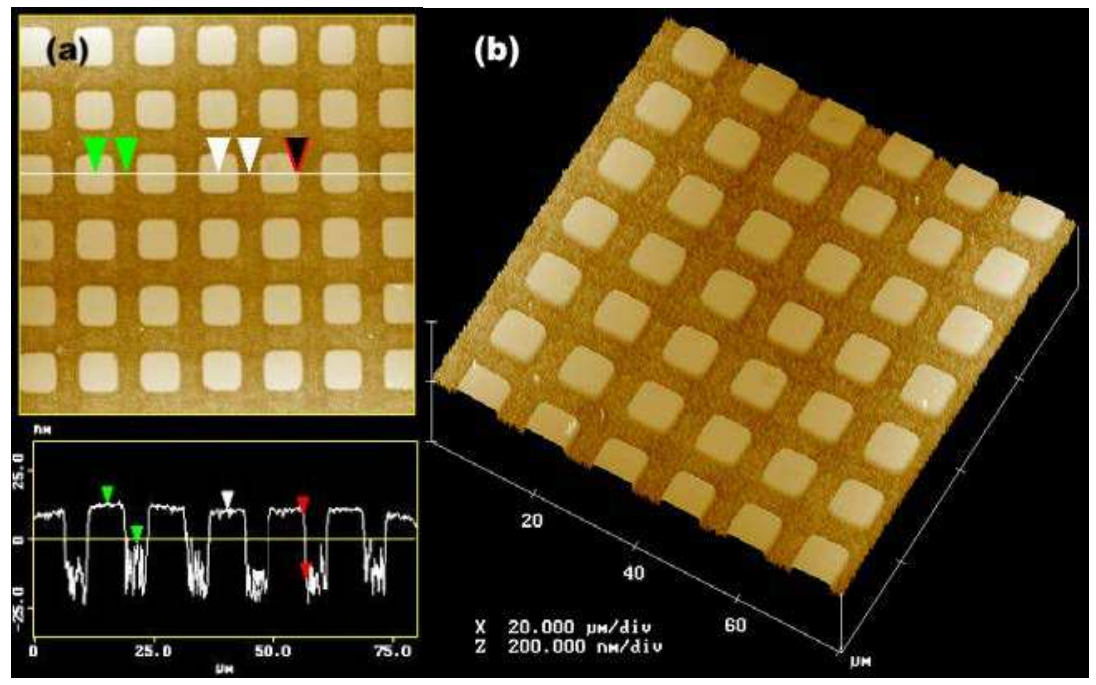

Fig. 5. Tapping-mode AFM images of periodic arrays of square silicon micromesa fabricated on $\mathrm{Si}(110)$ substrate with ODTS SAM (exposure time: $20 \mathrm{~min}$, etching time: $5 \mathrm{~min}$ ): (a) AFM image with sectional analysis of the arrays of silicon micromesas with a height of about 26 $\mathrm{nm}$; (b) 3D views of the arrays of silicon micromesa over a $80 \mathrm{um} \times 80 \mathrm{um}$ area and with a periodicity of $12.5 \mathrm{um}$. 


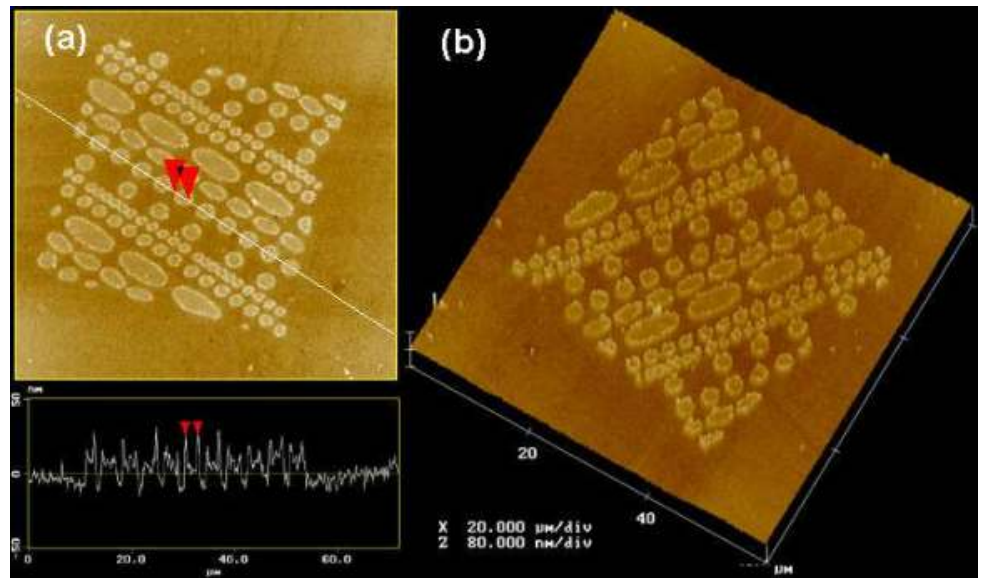

Fig. 6. Tapping-mode AFM images with sectional analysis of periodic arrays of square silicon microwell fabricated on $\mathrm{Si}(110)$ substrate with DDTS SAM (exposure time: 40min, etching time: 10min): (a) AFM images with sectional analysis of arrays of micromesas with a height of about $27 \mathrm{~nm}$; (b) 3D views of the arrays of silicon micromesas over a $60 \mathrm{um} \times 60$ um area.

When a TEM holey carbon grid was used as a mask, an interesting positive patterning result was obtained. The AFM images of the microstructures fabricated on the $\operatorname{Si}(110)$ substrate with DDTS SAM were displayed in figure 6, which corresponding to the positive patterning (exposure time: $30 \mathrm{~min}$; etching time: $10 \mathrm{~min}$ ). The rough micromesa with different diameters can be observed clearly in figure 6(a) and 6(b).

\subsection{Arrays of $\mathrm{Si}(100)$ microstructures}

Compared with the negative patterning shown in figure 5, a positive patterning could be obtained by adjusting the dosage of $\mathrm{He}^{*}-\mathrm{MAB}$ irradiation and the length of alkyl chains of SAM molecule. The AFM images of the square microwell arrays fabricated on the $\operatorname{Si}(100)$ substrate with DDTS SAM was shown in figure 7, corresponding to the positive patterning (exposure time: $20 \mathrm{~min}$; etching time: $10 \mathrm{~min}$ ). A square microwell arrays with a microwell size of $\sim 7 \times 7 \mu \mathrm{m}^{2}$ was fabricated on the $\mathrm{Si}(100)$ substrate. The depth of the microwells was about $220 \mathrm{~nm}$. The sharpest wall edge had a width of 1 pixel $(41 \mathrm{~nm})$ and a depth of $100 \mathrm{~nm}$, indicating a steep sidewall with an aspect ratio of 2.5:1. The sectional analysis shown in figure 7(a) indicated that the rms roughness of the bottom area of the microwell and the top area of the wall were $31 \mathrm{~nm}$ and $22 \mathrm{~nm}$, respectively. The rough characteristic could also be attributed to the imperfect SAM formation on the silicon substrate, as mentioned in 3.2. The positive-tone sensitivity of the SAM should be mainly due to the loss of hydrophobicity of DDTS SAM (with shorter alkyl chains, 12C) induced by the irradiation of the metastable helium atoms. The topography and line profile of the microwells array indicated a much rougher top surface of the sidewall with an rms roughness of $22 \mathrm{~nm}$. This large rms value suggested that the hydrophobic area might be much weaker in resisting the $\mathrm{KOH}$ etchant, and the DDTS SAM might not be a good resist for atom lithography. The corresponding 3D views of the array wae given in figure 7(b). Additionally, we also used TEM holey carbon grid as a mask to obtain a positive pattern result. The AFM images of the microstructures fabricated on the $\mathrm{Si}(100)$ substrate with HTS SAM was shown in figure 8, corresponding to 
the positive patterning. The rough microwell with different diameters can also be observed clearly in figure 8(a) and 8(b).

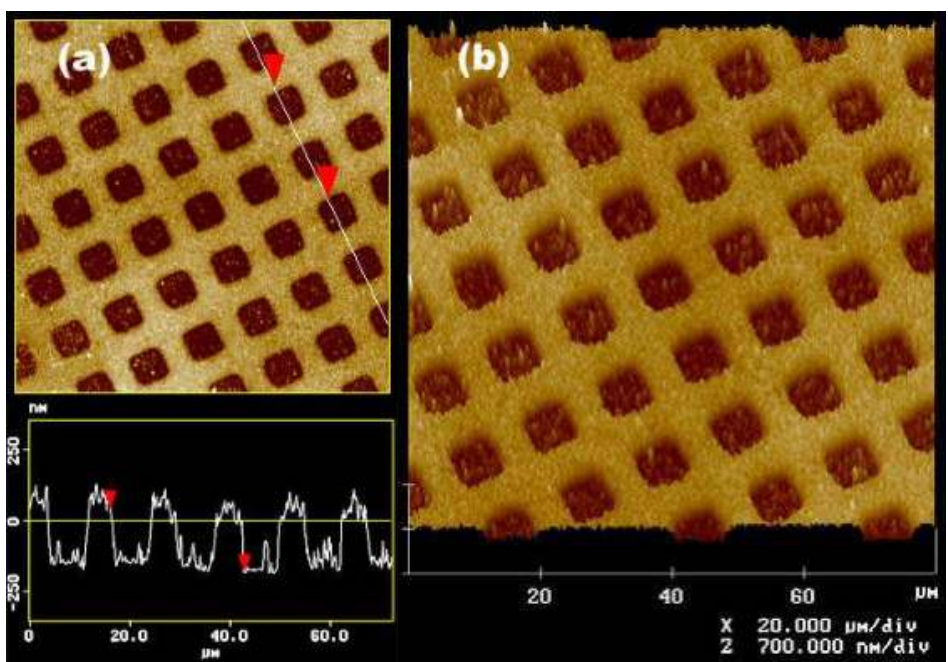

Fig. 7. Tapping-mode AFM images with sectional analysis of periodic arrays of square silicon microwell fabricated on $\mathrm{Si}(100)$ substrate with DDTS SAM (exposure time: 20min, etching time: 10min): (a) AFM images with sectional analysis of arrays of square microwell with a depth of about $220 \mathrm{~nm}$; (b) 3D views of the arrays of silicon microwell over a 80 um $\times 80$ um area and with a periodicity of $12.5 \mathrm{um}$.

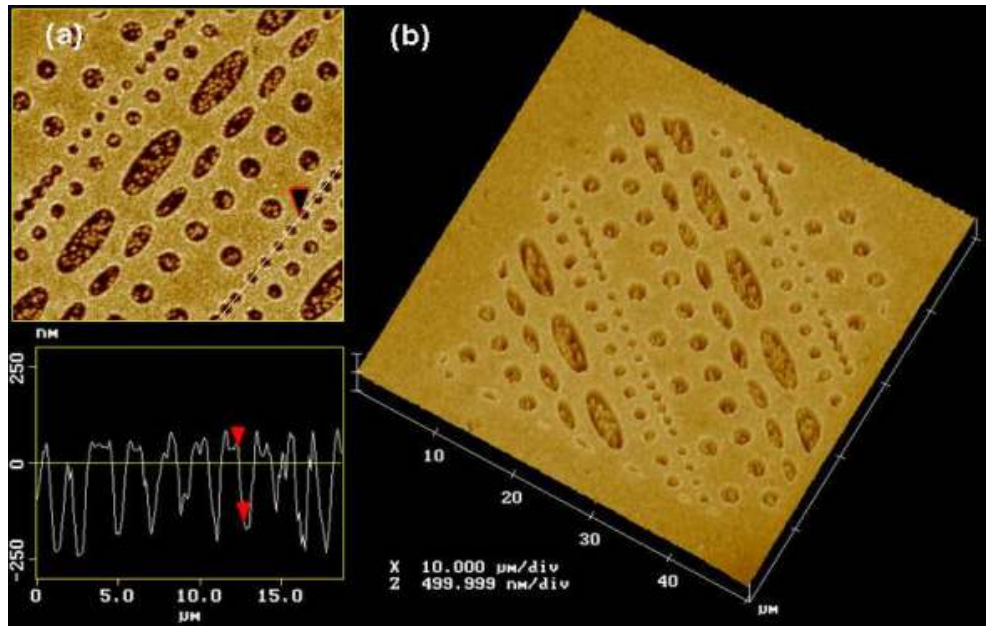

Fig. 8. Tapping-mode AFM images with sectional analysis of periodic arrays of square silicon microwell fabricated on $\mathrm{Si}(100)$ substrate with HTS SAM (exposure time: $30 \mathrm{~min}$, etching time: 10min): (a) AFM images with sectional analysis of arrays of square microwell with a depth of about $220 \mathrm{~nm}$; (b) 3D views of the arrays of silicon microwell over a 50 um $\times 50$ um area. 
The anisotropy of etching rate of silicon induced obvious difference of the spatial resolution of patterning of Si along different crystal orientation. In our experiments, the anisotropic etching rate seemed to improve the edge resolution, and the anisotropic etching process of silicon strongly affected the patterning results including the edge step resolution. It was easier to obtain a large aspect ratio and a better resolution for $\mathrm{Si}(110)$ rather than for $\mathrm{Si}(100)$. The sharpest step edge resolution obtained presently in $\mathrm{Si}(110)$ samples was only $41 \mathrm{~nm}$ with 1 pixel and the step edge resolution of about $120 \mathrm{~nm}$, indicating an aspect ratio of 3:1. In principle, the estimated value of geometrical blurring was $\sim 240 \mathrm{~nm}$ with the geometry described in scheme 3, and the spatial resolution was improved by reducing the distance between the source and the sample, which was related to the divergence of the metastable helium beam. The achievable width in our experiments was much smaller than the calculated one because of the hardening effect in the process of chemical etching. If the experimental parameters in both the exposure and etching processes (e.g., the exposure time, etching time, etc.) were optimized further, higher resolution of the patterning onto the silicon substrates could be obtained.

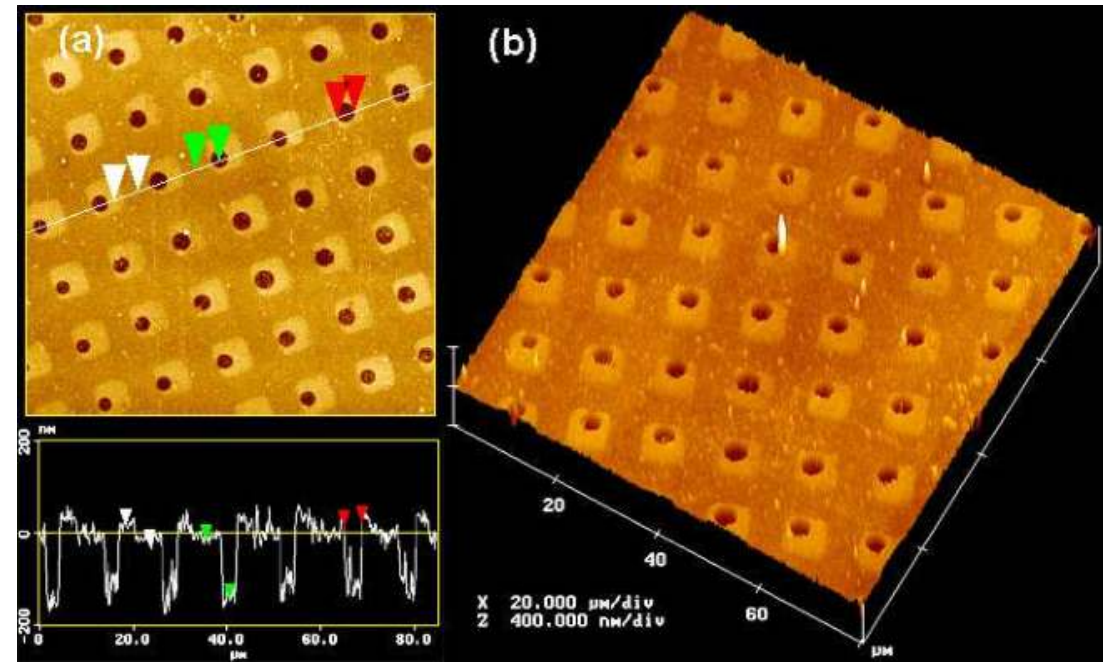

Fig. 9. Tapping-mode AFM images with sectional analysis of periodic arrays of square silicon microwell fabricated on $\mathrm{Si}(100)$ substrate with DDTS SAM (exposure time: 20min, etching time: 10min): (a) AFM images with sectional analysis of arrays of square microwell with a depth of about $180 \mathrm{~nm}$; (b) 3D views of the arrays of silicon microwell over a 80 $\mathrm{um} \times 80 \mathrm{um}$ area and with a periodicity of $12.5 \mathrm{um}$.

Athough the interaction between the outermost surface of the SAM and the irradiation of metstable atoms has been investigated for about ten years [34-41], the detailed change in surface chemistry of the outermost of the SAM molecules under the irradiation of helium atom beam is still difficult to predict and remains to be explored. What we demonstrated in the present study was that both the positive-tone and the negative-tone sensitivity of the SAM were related to largely different dosage of MAB and the length of the alkyl chains of SAM molecule (as illustrated in scheme 1 (IV) and (V)). When we optimized the experimental parameters, we accidentally obtained a novel patterning with both negative 
and positive pattern on one Si(100) substrate with DDTS SAM (as discribed in figure 9), which was very different from previous results. In this case, a novel array of square micromesas with microholes in it was fabricated successfully. The mechanism to induce this dual patterning is remained to be explored at present. This dual array also indicated some interesting flexibility of atom lithography in micro- and nanofabrication.

\section{Problems and perspectives}

Atom lithography based on $\mathrm{He}^{*}-\mathrm{MAB}$ and SAMs has been demonstrated to have significant potential in fabricating arrays of micro- and nanostructures. This new fabrication technique has opened a novel way in the practical application of the atom lithography in micro- and nanofabrication of silicon. Atom lithography using $\mathrm{He}^{*}-\mathrm{MAB}$ and SAMs to pattern the surface of silicon wafer without coating intermediate layer to create the arrays of silicon microstructures has been realized successfully. In order to improve the spatial resolution of the patterning, new etching method instead of $\mathrm{KOH}$ wet etching method need to be developed to meet the requirments of nanopaterning. At present, though the image of a pattern is in micrometers range due to TEM grids, a sharp edge in the tens-of-nanometers range indicates a potential of submicrometer-sized patterning. The new masks with nanopattern instead of present TEM masks should be used in order to generate nanostructure arrays. The mechanism of positive and negative patterning on silicon wafer has been investigated. Our results suggest that both the positive-tone and the negative-tone sensitivity of the SAM are related to largely different dosage of MAB and the length of the alkyl chains of SAM molecule. More experiments need to be carried out in order to conclude the detailed parameters to alter patterning type.

In the future, further studies on this subject can be listed as following:

1. To prepare more perfect SAMs for atom lithography;

2. To make new mask with sub-100nm pattern for patterning transfer;

3. To improve the precision to position and adjust the mask during exposing;

4. To investigate both other substrates (e.g. GaAs) and other etching processing (e.g. dry etching) for atom lithography.

\section{Acknowledgement}

The author gratefully acknowledges support from the National Natural Science Foundation of China (Grant No. 20777072, 91023040), the Fundamental Research Funds for the Central Universities (Grant No. WK2030020018) and would like to thank Prof. Yasushi Yamauchi, Dr. Mitsunori Kurahashi for their help with experiments and their helpful comments. The author also notes that part of the work was completed while he was at the NIMS.

\section{References}

[1] Xia, Y.; Rogers, J. A.; Paul, K. E. and Whitesides, G. M. (1999). Unconventional methods for fabricating and patterning nanostructures. Chem. Rev. 99 1823-1848.

[2] Campbell, C. J.; Smoukov, S. K. M.; Bishop, K. J.; Baker, E. and Grzybowski, B. A. (2006). Direct printing of 3D and curvilinear micrometer-sized architectures into solid substrates with sub-micrometer resolution. Adv. Mater. 18 2004-2008. 
[3] Ahn, S. J.; Kaholek, M.; Lee, W. K.; LaMattina, B.; LaBean, T. H. and Zauscher, S. (2004). Surface-Initiated Polymerization on Nanopatterns fabricated by electron-beam lithography. Adv. Mater. 16 2141-2145

[4] Wu, N.; Pease, L. F. and Russel, W. B. (2006). Toward large-scale alignment of electrohydrodynamic patterning of Thin Polymer Films. Adv. Funct. Mater. 16 19921999.

[5] Palmer, A. J.; Baker, M. and Sang, R. T. (2006). Towards creation of iron nanodots using metastable atom lithography. Nanotechnol. 17 1166-1170.

[6] Sawada, S.; Masuda, Y.; Zhu, P. and Koumoto, K. (2006). Micropatterning of copper on a poly(ethylene terephthalate) substrate modified with a self-assembled monolayer. Langmuir 22 332-337.

[7] Harnett, C. K.; Satyalakshmi, K. M. and Craighead, H. G. (2000). Low-energy electronbeam patterning of amine-functionalized self-assembled monolayers. Appl. Phys. Lett. 76 2466-2468.

[8] Kan, J. A. V.; Bettiol, A. A. and Watt, F. (2006). Proton beam writing of 3D nanostructures in hydrogen silses quioxane. Nano Lett. 6 579-582.

[9] Saito, N.; Kadoya, Y.; Hayashi, K.; Sugimura, H. and Takai, O. (2003). Micropatterned 1alkene self-assembled monolayer on hydrogen-terminiated silicon by vacuum ultraviolet lithography. Jpn. J. Appl. Phys. 42 2534-2537.

[10] Berggren, K. K.; Bard, A.; Wilbur, J. L.; Gillaspy, J. D.; Helg, A. G.; McClelland, J. J.; Rolston, S. L.; Phillips, W. D.; Prentiss, M. and Whitesides, G. M. (1995). Microlithography using neutral metastable atoms and self- assembled monolayers. Science 269 1255-1257.

[11] Dong, B.; Lu, N.; Zelsmann, M.; Kehagias, N.; Fuchs, H.; Torres, C. M. S. and Chi, L. (2006). Fabrication of high-density, large-area conducting-polymer nanostructures. Adv. Funct. Mater. 16 1937-1942.

[12] Jang, J. H.; Ullal, C. K.; Maldovan, M.; Gorishnyy, T.; Kooi, S.; Koh, C. Y. and Thomas, E. L. (2007). 3D micro- and nanostructures via interference lithography. Adv. Mater. 17 3027-3041.

[13] Henzie, J.; Lee, M. H. and Odom, T. W. (2007). Multiscale patterning of plasmonic metamaterials. Nature Nanotech. 2 549-554.

[14] Tao, A.; Sinsermsuksakul, P. and Yang, P. D. (2006). Tunable plasmonic lattices of silver nanocrystals. Nature Nanotech. 2 435-440.

[15] Lei, Y.; Cai, W. P. and Wilde, G. (2007). Highly ordered nanostructures with tunable size, shape and properties: A new way to surface nano-patterning using ultra-thin alumina masks. Progress in Materials Science 52 465-539.

[16] Shin, S. W.; Lee, S. G.; Lee, J.; Whang, C. N.; Lee, J. H.; Choi, I. H.; Kim, T. G. and Song, J. H. (2005). Ion-beam nano-patterning by using porous anodic alumina as a mask. Nanotechnol. 16 1392-1395.

[17] Tseng, A. A. (2005). Recent developments in nanofabrication using focused ion beams. Small 1 924-939.

[18] Carr, D. W.; Lercel, M. J.; Whelan, C. S.; Craighead, H. G.; Seshadri, K. and Allara, D. L. (1997). High-selectivity pattern transfer processes for self-assembled monolayer electron-beam resists. J. Vac. Sci. Technol. B 15 1446-1450. 
[19] Zhang, J. W.; Kurahashi, M.; Suzuki, T.; Sun, X. and Yamauchi, Y. (2006). Microfabrication of silicon using self-assembled monolayer resist and metastable helium beam. Jpn. J. Appl. Phys. 45 8020-8023.

[20] Zhang, J. W. (2009). Fabricating arrays of $\mathrm{Si}(110) / \mathrm{Si}(100)$ microstructures by atom lithography using organosilane self-assembled monolayers. J. Phys.: Conf. Ser. 188 012008

[21] Hill, S. B.; Haich, C. A.; Dunning, F. B.; Walters, G. K.; McClell, J. J.; Celotta, R. J. and Craighead, H. G. (1999). Patterning of hydrogen-passivated $\operatorname{Si}(100)$ using $\operatorname{Ar}(3 \mathrm{P} 0,2)$ metastable atoms. Appl. Phys. Lett. 74 2239-2241.

[22] Younkin, R.; Berggren, K. K.; Johnson, K. S.; Prentiss, M.; Ralph, D. C. and Whitesides, G. M. (1997). Nanostructure fabrication in silicon using cesium to pattern a selfassembled monolayer. Appl. Phys. Lett. 71 1261-1263.

[23] Lu, W.; Baldwin, K. G. H.; Hoogerland, M. D.; Buckman, S. J.; Senden, T. J.; Sheridan, T. E. and Boswell, R. W. (1998). Sharp edged silicon structures generated using atom lithography with metastable helium atoms. J. Vac. Sci. Technol. B 16 3846-3849.

[24] O’Dwyer, C.; Gay, G.; Viaris, de Lesegno B.; Weiner, J.; Camposeo, A.; Tantussi, F.; Fuso, F.; Allegrini, M. and Arimondo, E. (2005). Atomic nanolithography patterning of submicron features: writing an organic self-assembled monolayer with cold, bright Cs atoms beams. Nanotechnol. 16 1536-1541.

[25] Ju, X.; Kurahashi, M.; Suzuki, T. and Yamauchi, Y. (2004). Positive and negative patterning of ethanethiol, decanethiol, and hexadecanethiol self-assembled monolayers by using a metastabl helium beam. Thin Solid Films 464 420-424.

[26] Wang Z. P.; Kurahashi M.; Suzuki T.; Ding Z. J. and Yamauchi Y. (2010). Silicon micro/nanofabrication using metastable helium atom beam lithography. J. Nanosci. Nanotechnol. 10 7443-7446.

[27] Ju, X.; Kurahashi, M.; Suzuki, T. and Yamauchi, Y. (2005). Fabrication of gold patterns with nanoscale edge by using heptanethiol self-assembled monolayer and metastable helium beam. Appl. Surf. Sci. 241 241-245.

[28] Brezger, B.; Schulze, T.; Drodofsky, U.; Stuhler, J.; Nowak, S.; Pfau, T. and Mlynek, J. (1997). Nanolithography with neutral chromium and helium atoms. J. Vac. Sci. Technol. B 15 2905-2911.

[29] Hill, S. B.; Haich, C. A.; Dunning, F. B.; Walters, G. K.; McClelland, J. J.; Celotta, R. J.; Craighead, H. G.; Han, J. and Tanenbaum, D. M. (1999). Patterning of octadecylsiloxane self-assembled monolayers on $\operatorname{Si}(100)$ using $\operatorname{Ar}\left({ }^{3} \mathrm{P}_{0,2}\right)$ atoms. J. Vac. Sci. Technol. B 17 1087-1089.

[30] Kulkarni, S. A.; Mirji, S. A.; Mandale, A. B.; Gupta, R. P. and Vijayamohanan, K. P. (2005). Growth kinetics and thermodynamic stability of octadecyltrichlorosilane self-assembled monolayer on Si (100) substrate. Mater. Lett. 59 3890-3895.

[31] Shirahata, N.; Masuda, Y.; Yonezawa, T. and Koumoto, K. (2004). Atomic scale flattening of organosilane self-assembled monolayer and patterned tin hydroxide thin films. Eur. Ceram. Soc. 24 427-434.

[32] Heid, S.; Effenberger, F.; Bierbaum K. and Grunze M. (1996). Self-assembled mono- and multilayers of terminally functionalized organosilyl compounds on Silicon substrates. Langmuir 12 2118-2120.

[33] Sato, K.; Shikida, M.; Matsushima, Y.; Yamashiro, T.; Asaumi, K.; Iriye, Y. and Yamamoto, M. (1998). Characterization of orientation-dependent etching 
properties of single-crystal silicon: effects of $\mathrm{KOH}$ concentration. Sens. and Actuators A 64 87-93.

[34] Yamauchi, Y.; Noro, T.; Kurahashi, M.; Suzuki, T. and Ju, X. (2005). Metastable-atomstimulated Desorption from Dodecanethiolate Self-assembled Monolayer. Appl. Surf. Sci. 241 141-145.

[35] Setoyama, H.; Kera, S.; Okudaira, K. K.; Hara, M.; Harada, Y. and Ueno, N. (2003). Outermost surface reactions of molecular thin films induced by metastable-atom impacts. Jpn. J. Appl. Phys. 42 597-601.

[36] Thywissen, J. H.; Johnson, K. S.; Dekker, N. H.; Prentiss, M.; Wong, S. S.; Weiss, K. and Grunze, M. (1998). Metastable-atom-activated growth of an ultrathin carbonaceous resist for reactive ion etching of $\mathrm{SiO}_{2}$ and $\mathrm{Si}_{3} \mathrm{~N}_{4}$. J. Vac. Sci. Technol. B 16 1155-1160.

[37] Close, J. D.; Baldwin, K. G. H.; Hoffmann, K. and Quaas, N. (2000). Fragmentation of dodecanethiol molecules: application to self-assembled monolayer damage in atom lithography. Appl. Phys. B 70 651-655.

[38] Nowak, S.; Pfau, T. and Mlynek, J. (1996). Nanolithography with metastable helium. Appl. Phys. B 63 203-205.

[39] Johnson, K. S.; Thywissen, J. H.; Dekker, N. H.; Berggren, K. K.; Chu, A. P.; Younkin, R. and Prentiss, M. (1998). Localization of metastable atom beams with optical standing waves: nanolithography at the heisenberg limit. Science 280 1583-1586.

[40] Johnson, K. S.; Berggren, K. K.; Black, A. T.; Chu, A. P.; Dekker, N. H.; Ralph, D. C.; Thywissen, J. H.; Younkin, R.; Thinkham, M.; Prentiss, M. and Whitesides, G. M. (1996). Using neutral metastable argon atoms and contamination lithography to form nanostructure in silicon,silicon dioxide, and gold. Appl. Phys. Lett. 69 27732775.

[41] Rehse, S. J.; Glueck, A. D.; Lee, S. A.; Goulakov, A. B.; Menoni, C. S.; Ralph, D. C.; Johnson, K. S. and Prentiss, M. (1997). Nanolithography with metastable neon atoms:Enhanced rate of contamination resist formation for nanostructure fabrication. Appl. Phys. Lett. 71 1427-1429. 


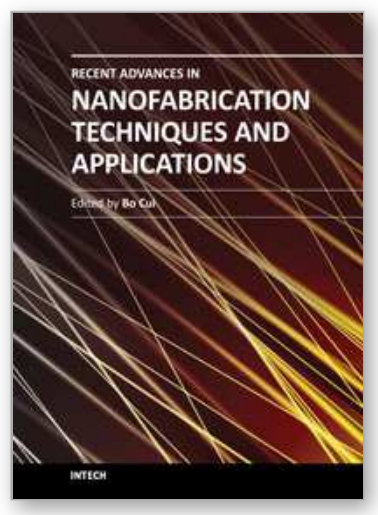

\author{
Recent Advances in Nanofabrication Techniques and Applications \\ Edited by Prof. Bo Cui
}

ISBN 978-953-307-602-7

Hard cover, 614 pages

Publisher InTech

Published online 02, December, 2011

Published in print edition December, 2011

Nanotechnology has experienced a rapid growth in the past decade, largely owing to the rapid advances in nanofabrication techniques employed to fabricate nano-devices. Nanofabrication can be divided into two categories: "bottom up" approach using chemical synthesis or self assembly, and "top down" approach using nanolithography, thin film deposition and etching techniques. Both topics are covered, though with a focus on the second category. This book contains twenty nine chapters and aims to provide the fundamentals and recent advances of nanofabrication techniques, as well as its device applications. Most chapters focus on indepth studies of a particular research field, and are thus targeted for researchers, though some chapters focus on the basics of lithographic techniques accessible for upper year undergraduate students. Divided into five parts, this book covers electron beam, focused ion beam, nanoimprint, deep and extreme UV, X-ray, scanning probe, interference, two-photon, and nanosphere lithography.

\title{
How to reference
}

In order to correctly reference this scholarly work, feel free to copy and paste the following:

Jianwu Zhang, Zhongping Wang and Zengming Zhang (2011). Atom Lithography: Fabricating Arrays of Silicon Microstructures Using Self-Assembled Monolayer Resist and Metastable Helium Beam, Recent Advances in Nanofabrication Techniques and Applications, Prof. Bo Cui (Ed.), ISBN: 978-953-307-602-7, InTech, Available from: http://www.intechopen.com/books/recent-advances-in-nanofabrication-techniques-andapplications/atom-lithography-fabricating-arrays-of-silicon-microstructures-using-self-assembled-monolayerresist

\section{INTECH}

open science | open minds

\section{InTech Europe}

University Campus STeP Ri

Slavka Krautzeka 83/A

51000 Rijeka, Croatia

Phone: +385 (51) 770447

Fax: +385 (51) 686166

www.intechopen.com

\section{InTech China}

Unit 405, Office Block, Hotel Equatorial Shanghai

No.65, Yan An Road (West), Shanghai, 200040, China 中国上海市延安西路65号上海国际贵都大饭店办公楼 405 单元

Phone: +86-21-62489820

Fax: +86-21-62489821 
(C) 2011 The Author(s). Licensee IntechOpen. This is an open access article distributed under the terms of the Creative Commons Attribution 3.0 License, which permits unrestricted use, distribution, and reproduction in any medium, provided the original work is properly cited. 